\title{
Pre-Operative Auditory-Perceptual Voice Characteristics in Patients Undergoing Thyroid and/or Parathyroid Surgery
}

\author{
Safinaz Nagib Azab ${ }^{1 *}$, Rasha A Alshareef ${ }^{2}$, Heba Abd Allah Abd Elmaged ${ }^{3}$ and Gamal Youssef ${ }^{4}$ \\ ${ }^{1}$ Assistant Professor of Phoniatrics, Faculty of Medicine, Beni Suef University, Egypt \\ ${ }^{2}$ Lecturer of Phoniatrics, Faculty of Medicine, Beni Suef University, Egypt \\ ${ }^{3}$ Phoniatrics Specialist, General Hospital, Beni Suef, Egypt \\ ${ }^{4}$ Assistant Professor of Phoniatrics, Faculty of Medicine, Alexandria, Egypt \\ *Corresponding author: Safinaz Nagib Azab, Unit of Phoniatrics, Department of Otorhinolaryngology, Faculty of Medicine, Beni Suef, \\ Egypt
}

\begin{abstract}
Background: The human voice is one of the most important tools in human every day. Communication and voice were probably important to human communication even before language evolved. Voice Quality can be affected in patients with thyroid disease like, loss of vocal range, low speaking fundamental frequency (especially in women), coarse and gravelly vocal symptoms, weak voice, and breathy quality, reduced Intensity and changes of the singing voice.

Purpose: The aim of this work is to identify auditory perceptual voice changes and Severity in patients with thyroid and/or parathyroid disease preoperatively.

Methods: 60 adult subjects their age ranged between 20 and 60 years old, both males and females, divided into two groups were included (Group A): 30 patients diagnosed with thyroid and/or parathyroid Disease preoperatively. (Group B): 30 healthy normal subjects. Both groups were undergone to Modified GRBAS scale, Voice Recording, and Flexible nasofibrolaryngoscopy.

Results: There is difference between the two groups regarding the presence or absence of dysphonia, chewing and swallowing, and parameters of APA (auditory perceptual assessment) except register.

Conclusion: The perceptual measure of voice by modified GRBAS, revealed significant differences in (overall grade of dysphonia, pitch, and loudness, of voice) between the individuals with thyroid disease and those in an age and gender-matched control group. If surgeons can screen for pre-surgical dysphonia, the rate of indeterminate postsurgical dysphonia may be reduced, or at least explained and prepared for.
\end{abstract}

Keywords: Voice parameters; thyroid diseases; pre-operative assessment

\section{Introduction}

The human voice is one of the most important tools in human everyday communication and the voice was probably important to human communication even before language evolved. Also, long before the infant develops language; it expresses important information by means of its voice organ [1]. Voice Quality can be affected in patients with thyroid disease. There are number of voice problems occur in thyroid disease including loss of vocal range, low speaking fundamental frequency (especially in women), coarse and gravelly vocal symptoms, weak voice, breathy quality, reduced. Intensity and changes of the singing voice [2]. Vocal impairment in thyroid disease may be related to structural or 'functional changes in the larynx or as the thyroid gland is closely related to the larynx via both innervation and blood supply. However, the nerves and arteries of the thyroid and larynx are intertwined, thus damage to one structure could easily affect the other structure [3]. Thyroid disease may or may not have a noticeable impact on laryngeal 
functioning. If a patient develops massive tumor, compression of the vocal folds results in dysphonia, stridor and dyspnea. When cervical nodes metastasize, vocal fold paralysis can occur related diseases that often co-occur with nodules such as hypothyroidism and hyperthyroidism may impact the laryngeal mechanism [3]. Voice related outcomes were reported post-operatively in patients who received thyroid surgery.

Patients were tested prior to surgery and postoperatively with the Visipitch oscilloscope, an objective tool used for voice analysis, which measures pitch and amplitude over time. Patients were also given a questionnaire following surgery. Patients were considered to be "worse" if there was a decrease in maximum phonation time more than 2 seconds, an increase in perturbation greater than $.05 \%$, or a decrease in percentage voiced by $2 \%$. "Improvement" was judged by using the same guidelines in the opposite direction [4]. Patients may have voicing abnormalities before thyroid surgery is performed and surgery may improve or worsen the voice irrespective of the pre-operative voice status [4]. There is lack of information on the incidence of voice disorders in those with thyroid disease [5] reports the possibility of dysphonia, stridor and dyspnea in those with thyroid tumors. Hypothyroidism can cause perceived voice changes such as lower pitch, roughness, decreased range, and vocal fatigue; whereas hyperthyroidism can cause shaky voice, breathy vocal quality, and reduced intensity beside that the impact of aging on the larynx can also cause perceptual changes in the voice [3]. Modified GRBAS has been shown to be a reliable measure for identifying perceived vocal changes. The ease of use and high predictive value of this measure makes it ideal for measuring voice parameters as experienced by the listener [5]. If those with thyroid disease often display vocal dysfunction, detection of these symptoms could aid in early detection of thyroid disease. Further, obtaining a baseline for voice dysfunction in those with thyroid disease could facilitate documentation of the recovery process. Moreover, baseline data could help surgeons determine the success of their surgical techniques based on postoperative vocal function [6].

\section{Objectives}

The aim of this work is to identify auditory perceptual voice changes and Severity in patients with thyroid and/or parathyroid disease preoperatively.

\section{Subjects \& Methods}

This study included 60 adult subjects divided into two groups:

a) Group A: 30 patients diagnosed with thyroid and/or parathyroid Disease preoperatively

b) Group B: 30 healthy normal subjects their age ranged between 20 and 60 years old, both males and females. Both groups were matched in age and gender.

\section{Exclusion criteria: Applied on both groups}

a) Smoking, alcohol or drug abuse.

b) Those on any regular medication which might have an effect on voice quality. c) Those with respiratory tract infection on the day of assessments.

d) History of severe respiratory allergies, asthma, neuromotor impairment, hearing impairment, or psychiatric problems.

e) History of neck surgery in the past years.

f) History of misuse and abuse of voice.

g) Gross laryngeal lesions by laryngoscopy.

All patients were subjected to:

1) Protocol of voice assessment that was applied in phoniatrics unit Beni-Suef university hospital and in Alexandria University hospital.

2) Auditory perceptual assessment (APA) GRBAS modification: [7] described a modified scale for Evaluation of dysphonic voice which called a "modified GRBAS scale" where the followings were evaluated:

a) Overall grade: Normal (0), Slight (1), Moderate (2) or Severe (3).

b) Character (quality):

i. $\quad$ Strained (S): perception of excessive vocal effort.

ii. Leaky (L): excessive air escape during phonation under pressure.

iii. Breathy (B): excessive air escape during phonation not under pressure.

iv. Irregular or "rough" (I): lack of clear vocal quality.

c) Pitch: Overall increase, Overall decrease, Diplophonia

d) Register: Habitual, Modal or falsetto; Tendency to vocal fry at the end of phrase; Register break

e) Loudness: Excessively loud, Excessively soft, Fluctuating

f) Glottal attack: Normal, Soft, Hard

g) Associated Laryngeal Functions: Cough, Whisper, Laugher

Voice recording was carried out in a sound treated room, the patient was sitting $10 \mathrm{~cm}$ away from the microphone and Off-axis positioning (45-90 from the mouth axis) reduces aerodynamic noise from the mouth during speech. Regarding voice/speech material, examples of protocol for standard recording were as follows:

a. The patient counted to ten at comfortable pitch and loudness.

b. /a/: at (spontaneous) comfortable pitch/loudness, recorded three times to evaluate variability of quality.

c. /a/: slightly louder to evaluate the possible change in quality (plasticity)

d. A single sentence or a short standard passage Phonetic selection can be useful, such as a short sentence with constant 
voicing (no voiceless sounds and spoken without interruption) and no fricatives.

3) Indirect laryngoscope examination: The larynx was visualized simply by using laryngeal mirror. For assessment of vocal fold structure, configuration and gross mobility.

4) Flexible nasofibrolaryngoscopy: To visualize the vocal folds during various articulatory maneuvers and allows visualization not only the larynx but also of supra glottal structure and even velopharyngeal mechanism [8].

Statistical tests used in this thesis were:

a. Description of qualitative variables by frequency and percentage.

b. Description of quantitative variables in the form of mean and standard Deviation (mean $\pm \mathrm{SD}$ ).

c. Chi-square (x2) test was used for comparison of qualitative variables with each other.

a) Comparison between quantitative variables was carried by using: -Student t-test of two independent samples.
Significance level (p) was expressed as following:

a. P value $>0.05$ is insignificant (NS).

b. P value $<0.05$ is significant(S).

c. P value $<0.001$ is highly significant (HS).

\section{Results}

The study's sample included 60 subjects their ages ranged between (22 and 58 years) with a mean (36.5 \pm 8.6$)$ table (2), they were 8 males and 52 females and were divided into two groups (Group A): 30 patients diagnosed with thyroid and/or parathyroid disease preoperatively consisted of 4 males and 26 females their ages ranged between (23 and 58 years) with a mean $\pm \operatorname{SD}(36.8 \pm 8.5)$. (Group B): 30 healthy normal subjects consisted of 4 males and 26 females their ages ranged between (22 and 57 years) with a mean \pm SD (36.2 \pm 8.7$)$. Both groups were matched as regard age and gender.

\section{Results of Auditory Perceptual Assessment (APA) by} GRBAS modification

Figures 1-10.



Figure 1: Shows highly significant difference between the two groups (A\&B) regarding chewing and swallowing affection.

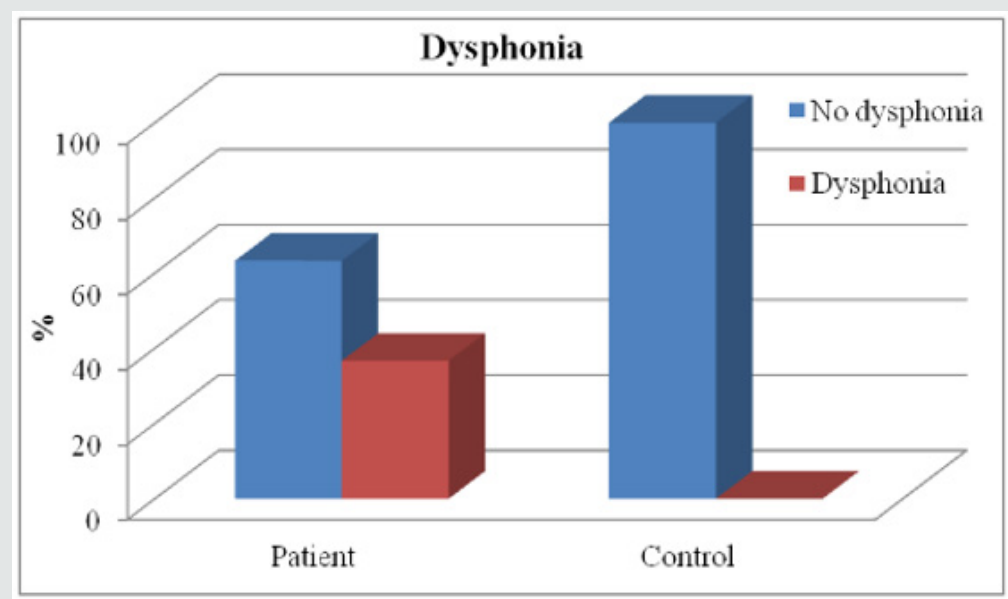

Figure 2: Shows highly significant difference between the two groups (A\&B) regarding dysphonia. 


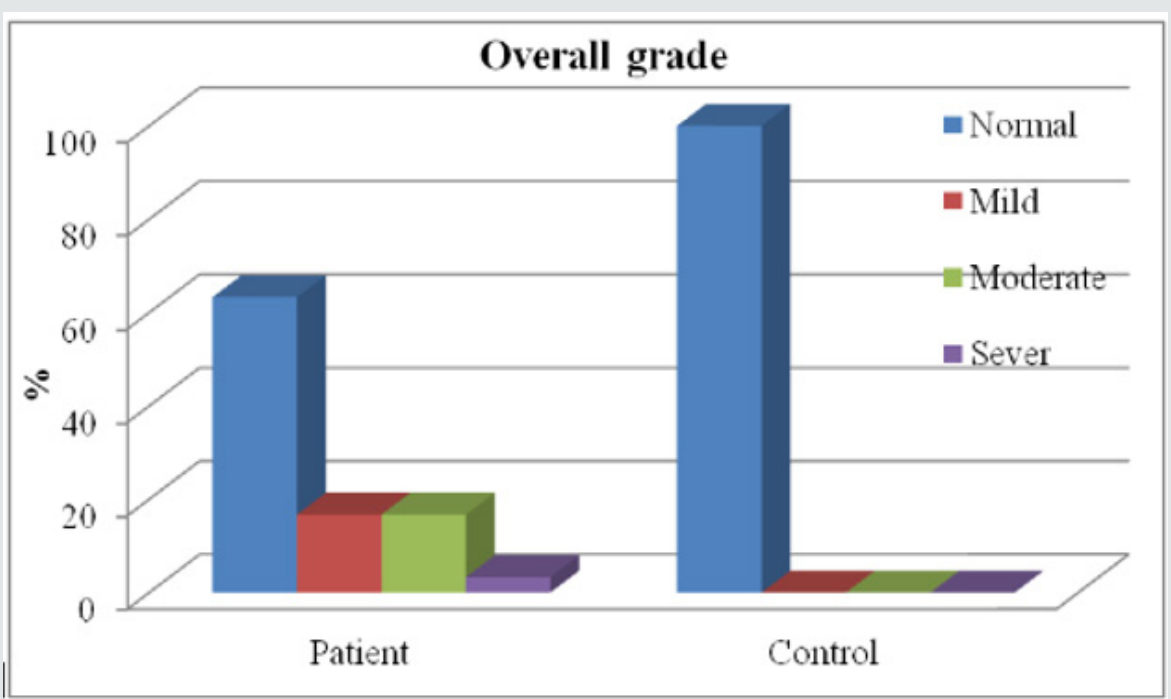

Figure 3: Shows highly significant difference between the two groups (A\&B) regarding overall grade.



Figure 4: Shows highly significant difference between the two groups (A\&B) regarding character.

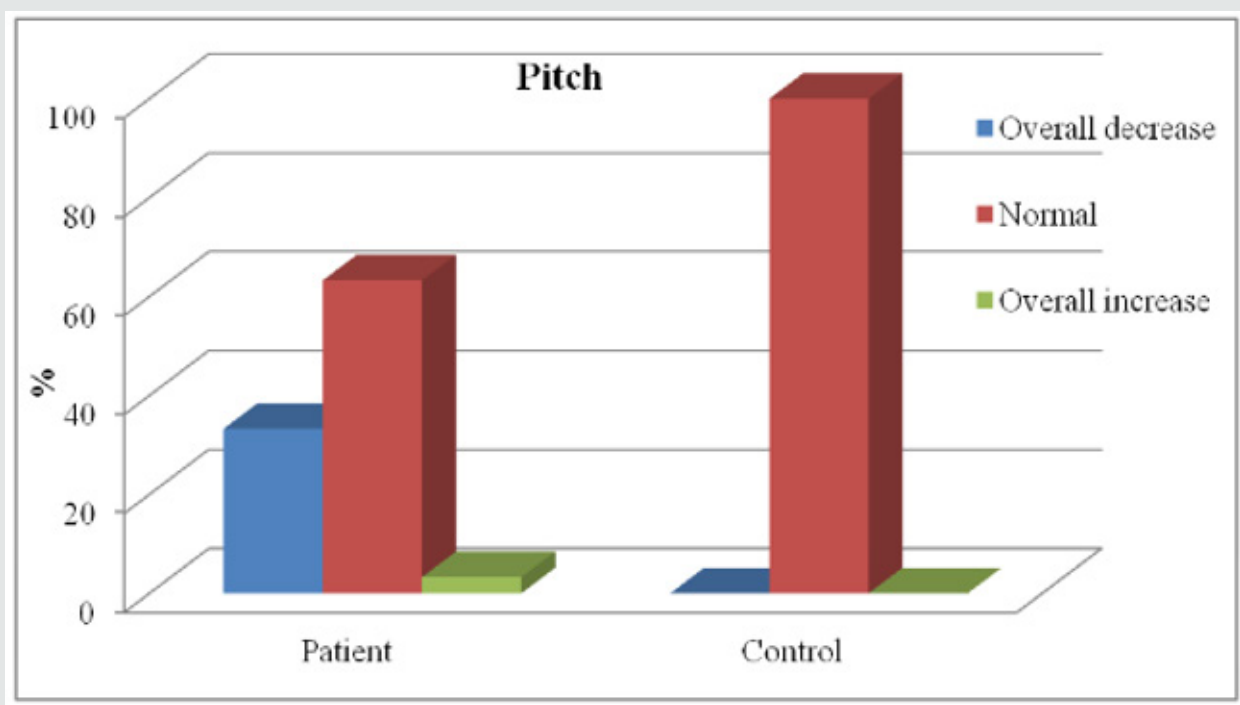

Figure 5: Shows highly significant difference between the two groups (A\&B) regarding pitch. 


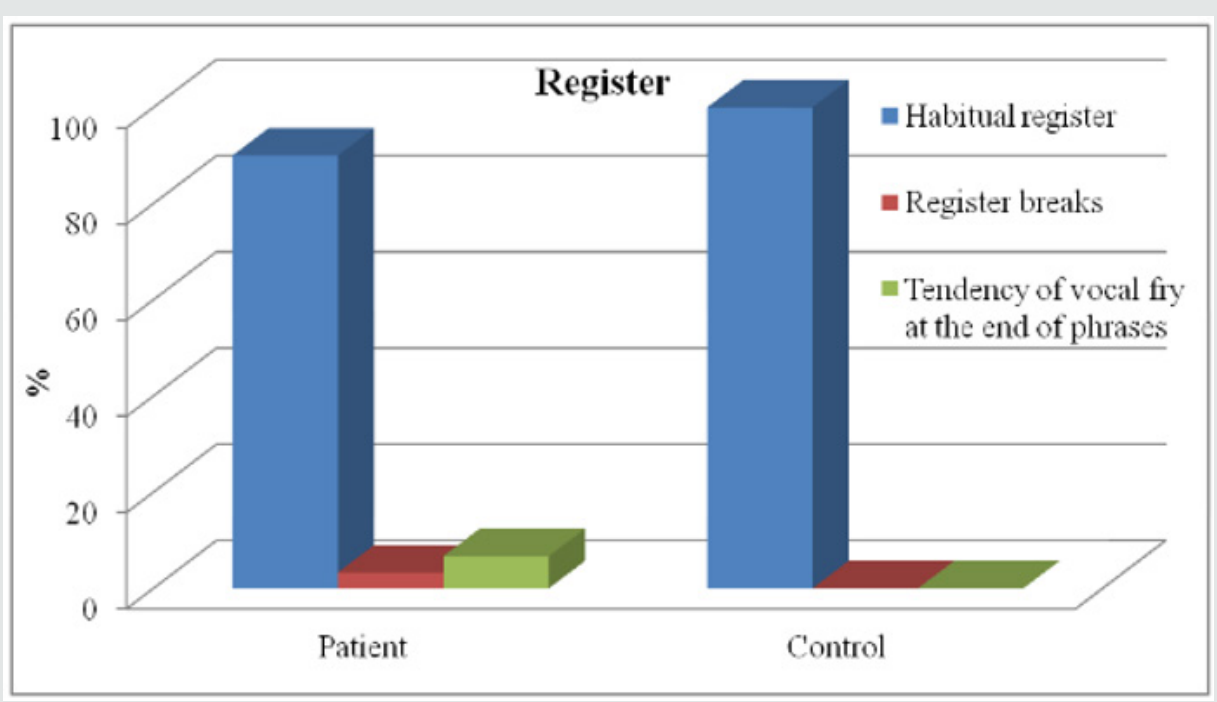

Figure 6: Shows no significant difference between the two groups (A\&B) regarding register.

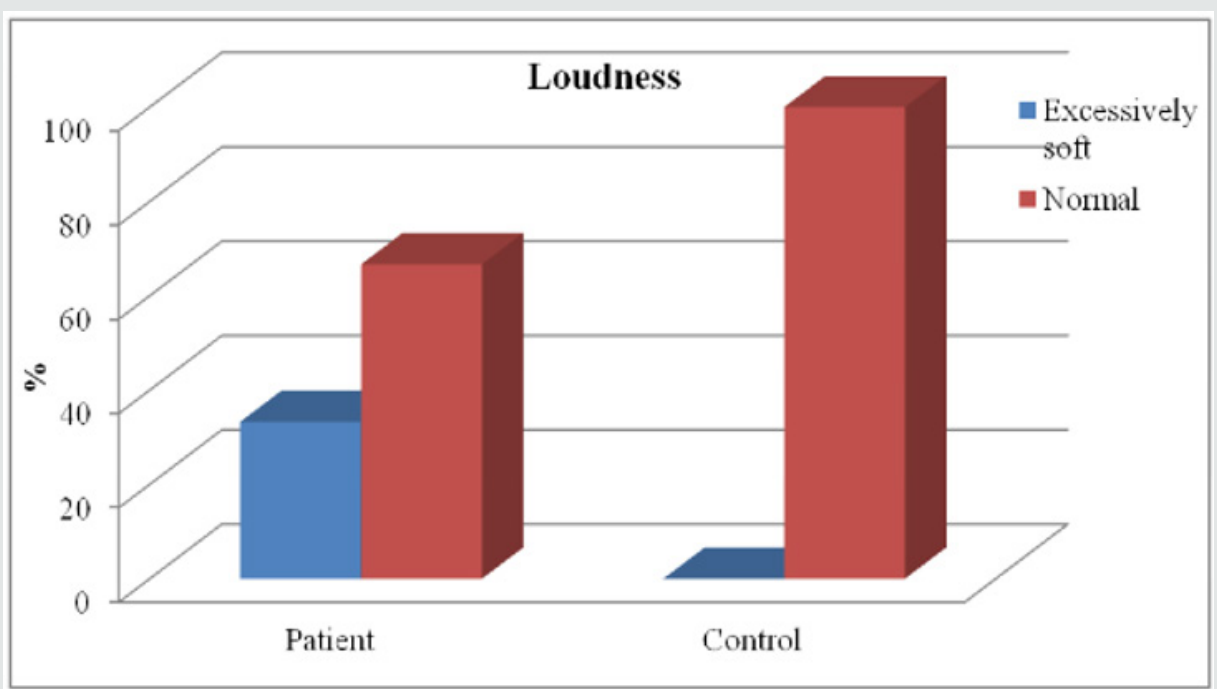

Figure 7: Shows highly significant difference between the two groups (A\&B) regarding loudness.

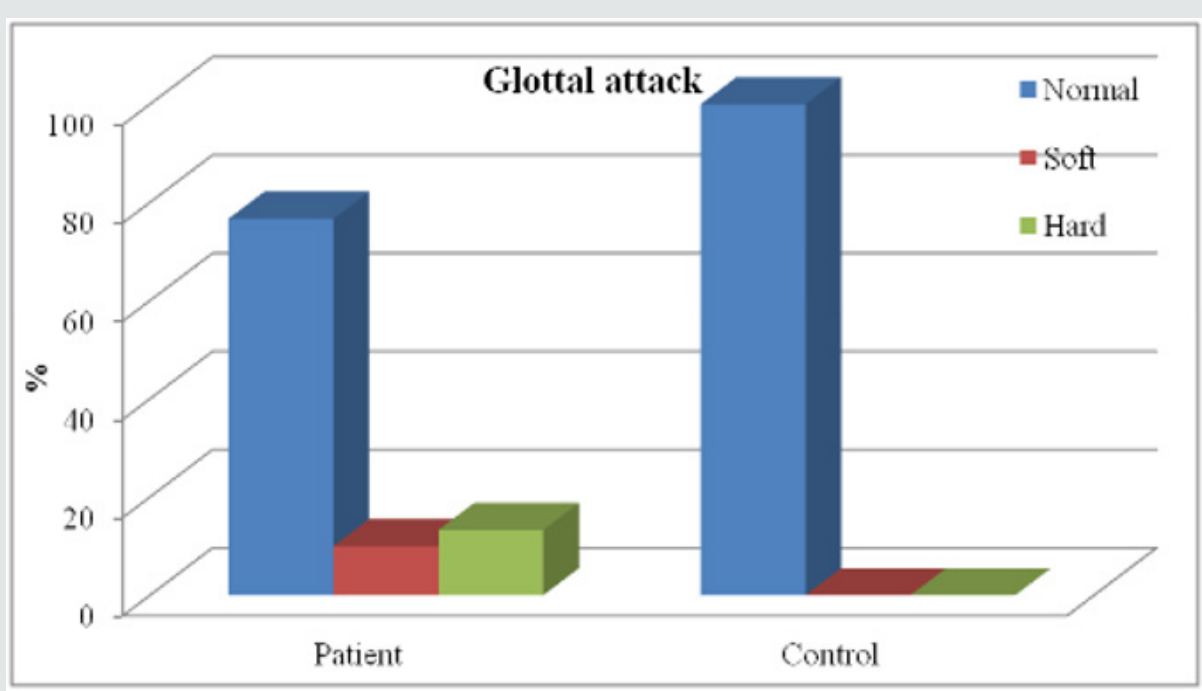

Figure 8: Shows significant difference between the two groups (A\&B) regarding glottal attack. 


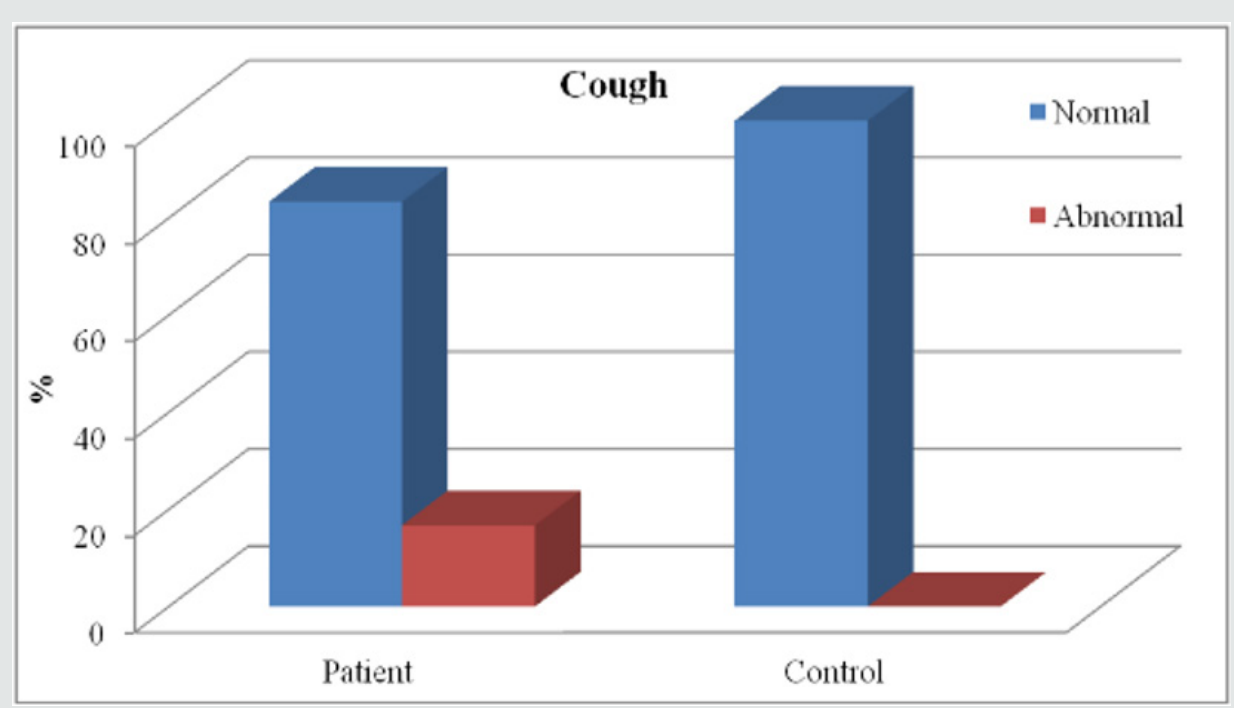

Figure 9: Shows significant difference between the two groups (A\&B) regarding cough.



Figure 10: Shows highly significant difference between the two groups (A\&B) regarding laughter.

\section{Discussion}

Voice is central to a wide range of human activities, and it's an audible sound by phonation which is the physical act of sound production by mean of vocal fold interaction with the exhaled air stream. A voice disorder exists when a person's quality, pitch and loudness of voice differ from those of persons of similar age, sex, cultural and geographic background [9]. Several studies had examined voice changes in patients following thyroidectomy, but there was a lack of studies that determined changes of vocal quality prior to thyroidectomy, Their for the purpose of our study was to identify auditory perceptual changes in voice for those with thyroid disease pre-operatively and determine how these changes differed from a control group. Results of this current study showed that $86.7 \%$ of thyroid patients were females and these results were in agreement with [10]. who suggested that the prevalence of thyroid disease is (3.0 to $9.0 \%$ ) of population with percentage ( $80 \%$ females and $20 \%$ males) which was explained by thyroid disease may be an autoimmune disease (Hashimoto thyroiditis),which is common in females with prevalence rate $4: 1$ female to male, also thyroid diseases are hormonal dependent so they are common during pregnancy. Also, these results were in agreement with [11]. They made a study involved an evaluation of 96 participants with thyroid or parathyroid disease who were referred to speech pathology by Otolaryngologists for a pre-operative voice assessment there were 72 (75\%) females and 24 (25\%) males. Results Showed highly significant difference between the two groups regarding chewing and swallowing affection, that $26.7 \%$ of patient group were complaining of chewing and swallowing disturbance with P-value (0.002). These results were in agreement with [12]. Who studied patients with thyroid disease pre-operatively and found that (25\%) of patients were complaining of swallowing disorders? Results of Auditory Perceptual Assessment (APA) by GRBAS.

Modification Showed highly significant difference between the two groups $(A \& B)$ regarding dysphonia, these results showed that 
$36.7 \%$ of patients with thyroid disease were dysphonic with P-value (0.001) and these results were in agreement with [4] who had a study which examined patients before and after thyroidectomy, they used Visipitch (Kay Pentax, Lincoln Park, NJ) to measure levels of dysphonia. They also used a subjective questionnaire after surgery to determine the patient's view on their voice. Onethird of their patients (15/44) presented with dysphonia before surgery and $22 \%$ reported voice abnormalities after. According to their findings, patient reported dysphonia matched the dysphonia recorded with Visipitch with only $64 \%$ accuracy. They also used a subjective questionnaire after surgery to determine the patient's perception of their voice. High significant difference between the two groups (A\&B) regarding the Grade of dysphonia were showed in the results which showed that $(16.7 \%)$ of patients with thyroid disease had mild degree of dysphonia, also (16.7\%) had moderate degree of dysphonia and ( $3.3 \%$ ) had sever degree of dysphonia. These results were also founded by [13]. Who found that (19.2\%) of patients with thyroid disease had mild degree of dysphonia, also $(14.1 \%)$ had moderate degree of dysphonia and (2.2\%) had sever degree of dysphonia? Explaining that the majority of cases about (89\%) diagnosed with thyroid disease being multinodular goiter, hypothyroidism, and thyroid nodule which had mild to moderate effect on vocal performance, however only (11\%) of cases diagnosed as cancer thyroid which usually infiltrating the vocal folds resulting in sever changes in vocal performance. In the current study patients with thyroid disease showed a high significant difference between them and the control group in the character of voice about one third of patients (11/30) showed changes in voice quality which became: ( breathy, strained or strained leaky), breathy voice was(3.3\%), strained voice was(10\%) and strained leaky voice was(23.3\%).

These results agree with the results obtained by [14]. In the study that involved an evaluation of 96 participants with thyroid disease who were referred to speech pathology by otolaryngologists for a pre-operative voice assessment. They found that $(29.6 \%)$ of cases with strained and/or leaky character and (5.3\%) of cases were breathy in character. There are many premorbid conditions which can affect the voice but are often disregarded in the context of dysphonia before a thyroidectomy procedure [15]. Performed a study which looked at different conditions that affected voice quality using the Dysphonia Severity Index (DSI). They found smoking had an effect on the DSI of patients. Their studies showed patients who had smoked had worse DSI scores and this remained true 6 months postoperatively. Their study also illustrated scores on the DSI tended to be predictive of voice abnormalities after surgery for the first 6 months. Patients who had smoked could potentially have a more resilient voice abnormality because they showed a worse score initially and a decreased score after surgery. Smoking and other forms of poor vocal hygiene can contribute to dysphonia. But the present study results disagree with results obtained by [16] and showed that the voice of patients were examined before surgery as well Laryngostroboscopy was used to visualize the vocal folds, the voices were recorded and the Multi-Dimensional Voice Program (Kay Pentax) was used for assessment. Showed no significant deterioration in the character of voice. This study used objective measurements in their data collection but did not assess the patients' or clinicians' opinions on the voices. Voice quality can be affected in patients with thyroid disease, including loss of vocal range, vocal fatigue, and low speaking fundamental frequency.

(Especially in women), hoarseness, coarse and gravelly vocal Symptoms, weak voice, breathy quality, reduced Intensity and changes to the singing voice [2]. Results showed that one third $(10 / 30)$ of patients with thyroid disease had low pitched voice. These results explained by [3] as hypothyroidism or underactive thyroid hormone production reduces tissue fluids throughout the body, This loss of fluid may affect the health and physiology of hair, skin, and organs .With respect to vocal symptoms, the larynx often loses fluid in the lamina propria, as the deeper layers experience edema, due to these physical changes patients may experience voice changes such as lower pitch. These results agree with the results obtained by [17] that performed a study of 395 patients which utilized pre-surgical data. Their study focused on subjectively reported dysphonia before the surgery, the patients and their families were presented with a questionnaire which asked for a perceptual assessment of the voice, whether it was normal or not classified based on hoarseness, low in pitch, or prone to fatigue. $30 \%$ of patients reported with voice abnormalities such as low-pitched voice and leaky voice. Results showed no significant difference between the two groups regarding register. These results agree with the results obtained by [18] that used a variety of methods to assess the vocal condition of patients with thyroid disease allowed for a more complete picture of the characteristics of a patient's voice.

Found that there were no changes in register. Results showed highly significant difference between the two groups regarding loudness with P-value (0.001) showed that one third (10/30) of patients with excessively soft loudness. These results agree with [4] they found that One-third of their patients presented with excessively soft loudness before surgery, also these results agree with $[18,19]$ They found that patients with thyroid dysfunction had excessively soft loudness. The changes in voice loudness explained by [3] that proved that hyperthyroidism changes affect voice stability and loudness. But the current study results didn't agree with results obtained by [14] that applied auditory perceptual assessment for 96 patients with thyroid disease pre-operatively and found that there were no significant changes in patients' voice loudness. Current study results showed significant difference between the two groups regarding glottal attack, showed that $(10 \%)$ of patients with thyroid with soft glottal attack, and (13.3\%) of patients with thyroid with hard glottal attack that with P-value (0.019), which disagree with results obtained by [14] who found that no significant changes in glottal attack with P value (0.932) using Kruskal-Wallis test which is a non-parametric test used to compare several independent groups. [20] evaluated patient-reported and clinician determined voice assessments for identifying post thyroidectomy dysphonia, Patients were evaluated using patient-reported symptoms (Voice Case History [VCH]), patient-perceived voice handicap (Voice Handicap Index [VHI]), clinician-perceived voice 
deficits (CAPE-V) and video laryngoscopy (VLS) . The purpose of the study was, "To examine the utility of patient reported and cliniciandetermined voice assessment in identifying post thyroidectomy voice dysfunction. Fifty military health beneficiaries scheduled to undergo thyroid resection were included in the study. Persons with preoperative laryngeal dysfunction or prior neck/thyroid surgery were excluded. Participants were evaluated preoperatively and 3 times postoperatively (1-2 weeks, 3 and 6 months). There are cases of postsurgical dysphonia which present without any damage to the laryngeal nerves.

Most of the literature on post-thyroidectomy dysphonia provides examples of dysphonia without damage to these nerves [16]. There are many other potential causes of postsurgical dysphonia: complications and bruising from endotracheal intubation, edema of the Laryngeal complex, trauma resulting from the surgery itself, localized neck pain, dysfunction of the cricothyroid muscle, psychogenic dysfunctions. Finally, the current results are going with our hypothesis that patients with thyroid disease have changes in vocal quality prior to thyroidectomy.

\section{Conclusion}

There continues to be a percentage of patients who undergo thyroidectomies and develop post-surgical vocal disturbances without any obvious causes, one consideration to explain these post-surgical rates of dysphonia is to determine if pre-surgical levels of dysphonia exist in these patients. If surgeons can screen for pre-surgical dysphonia, the rate of indeterminate postsurgical dysphonia may be reduced, or at least explained and prepared for. The perceptual measure of voice by modified GRBAS, revealed significant differences in (overall grade of dysphonia, pitch, loudness, register of voice) in a manner that can be considered as a sensitive and reliable tool of assessment.

\section{References}

1. Segre R (1971) Senescence of the Voice, Eye, Ear, Nose and Throat, Monthly 50: 223-227.

2. Soylu L, Ozbas S, Uslu HY, Kocak S (2007) The evaluation of the causes of subjective voice disturbances after thyroid surgery. Am J Surge 194: 317-322.

3. Stemple JC, Glaze L, Klaben B (2010) Clinical voice pathology: Theory and management. San Diego Plural Publishing.

4. McIvor NP, Flint DJ, Gillibrand J, Morton RP (2000) Thyroid surgery and voice related outcomes. Australian and New Zealand Journal of Surgery 70(3): 179-183.
5. Ramirez AT, Gibelli B, Tradati N, Giugliano G, Zurlo V, et al. (2007) Surgical management of thyroid cancer. Expert Review of Anticancer Therapy 7(9): 1203-1214.

6. Linville SE (2004) The aging voice The ASHA Leader.

7. Kotby MN (1986) Voice disorders: Recent diagnostic advances. Egyptian Journal of Otolaryngology 3(1): 69-98.

8. Colton RH, Casper JK (1990) Understanding voice problems. A physiological perspective for diagnosis and treatment. John P Butler (Eds.), Baltimore, USA Library of congress cataloging pp. 362-371.

9. Aronson AE (1990) Clinical Voice Disorders $3^{\text {rd }}$ (Edn.), George thieme Verlage, Stuttgart, New York, USA.

10. Galofré J, Lomvardias S, Davies T (2008) Evaluation and treatment of thyroid nodules: A clinical guide. Mount Sinai Journal of Medicine: A Journal of Translational and Personalized Medicine 75(3): 299-311.

11. Bone SL, Vertigan AE, Eisenberg RE (2011) Preoperative assessment of voice abnormalities in patients with thyroid disease: a clinical datamining exploration of 'thyroid voice'; in Giles R, Epstein I, Vertigan A (Eds.), Clinical Data Mining in an Allied Health Organization: A Real World Experience. Sydney, Sydney University Press, USA.

12. De Pedro Netto I, Fae A, Vartanian JG, Barros APB, Correia LM (2006) Voice and vocal self-assessment after thyroidectomy. Head \& Neck 28(12): 1106-1114.

13. Carding PN, Wilson JA, MacKenzie K, Deary IJ (2000) Measuring voice outcomes: state of the science review. J Laryngeal Otol 123: 823-829.

14. Henry LR, Helou LB, Solomon NP, Howard RS, Gurevich Uvena J, et al. (2010) Functional voice outcomes after thyroidectomy: an assessment of the Dysphonia Severity Index (DSI) after thyroidectomy. Surgery 147: 861-870.

15. Karnell MP, Melton SD, Childes JM, Coleman TC, Dailey SA (2007) Reliability of clinician-based (GRBAS and CAPE- $V$ ) and patient-based (V-RQOL and IPVI) documentation of voice disorders. J Voice 21: 576590.

16. Page C, Zaatar R, Strunski V (2007) Subjective voice assessment after thyroid surgery: A prospective study of 395 patients. Indian Journal of Medical Sciences 61: 448-454.

17. Meek P, Carding N, Howard DH, Lennard TW (2007) Voice change following thyroid and parathyroid surgery. J Voice 22: 765-772.

18. Birkent H, Karacalioglu O, Merati AL, Akcam T, Gerek M (2008) Prospective study of the impact of thyroid hormone replacement on objective voice parameters. Ann Otol Rhino Laryngeal 117: 523-527.

19. Stojadinovic A, Henry LR, Howard RS, Gurevich Uvena J, Makashay MJ (2008) Prospective trial of voice outcomes after thyroidectomy: Evaluation of patient-reported and clinician-determined voice assessments in identifying post thyroidectomy dysphonia. Surgery 143(6): 732-742. 
This work is licensed under Creative Commons Attribution 4.0 License

To Submit Your Article Click Here: Submit Article

DOI: $10.32474 /$ SJ0.2020.04.000185

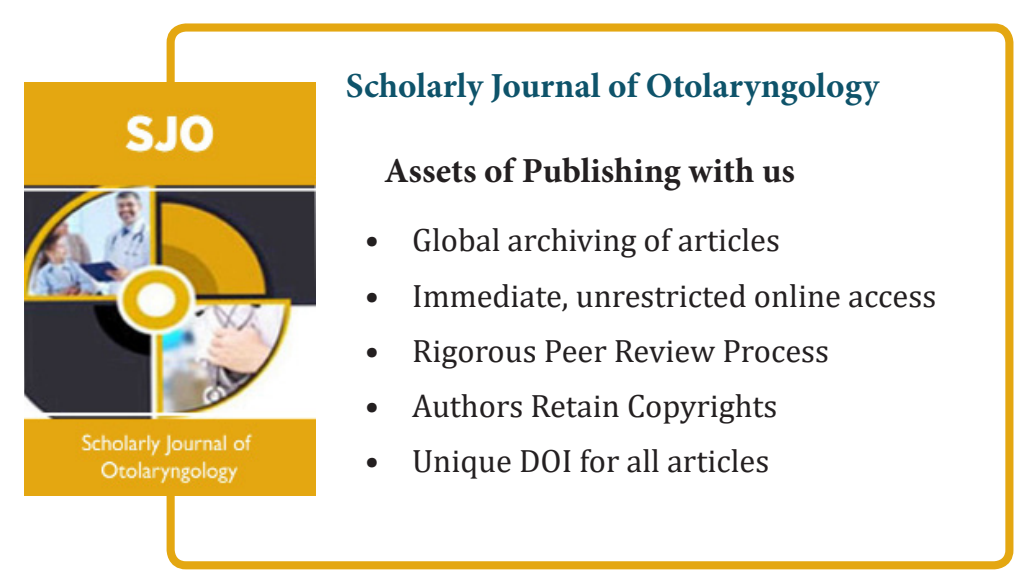

